\title{
Non-radial solar wind flows induced by the motion of interplanetary coronal mass ejections
}

\author{
M. Owens ${ }^{1, *}$ and P. Cargill ${ }^{1}$ \\ ${ }^{1}$ Space and Atmospheric Physics, The Blackett Laboratory Imperial College, London SW7 2BW, UK \\ *now at: Center for Space Physics Boston University, Boston MA 02215, USA
}

Received: 22 December 2003 - Revised: 28 August 2004 - Accepted: 3 September 2004 - Published: 22 December 2004

\begin{abstract}
A survey of the non-radial flows (NRFs) during nearly five years of interplanetary observations revealed the average non-radial speed of the solar wind flows to be $\sim 30 \mathrm{~km} / \mathrm{s}$, with approximately one-half of the large ( $>100 \mathrm{~km} / \mathrm{s}$ ) NRFs associated with ICMEs. Conversely, the average non-radial flow speed upstream of all ICMEs is $\sim 100 \mathrm{~km} / \mathrm{s}$, with just over one-third preceded by large NRFs. These upstream flow deflections are analysed in the context of the large-scale structure of the driving ICME. We chose 5 magnetic clouds with relatively uncomplicated upstream flow deflections. Using variance analysis it was possible to infer the local axis orientation, and to qualitatively estimate the point of interception of the spacecraft with the ICME. For all 5 events the observed upstream flows were in agreement with the point of interception predicted by variance analysis. Thus we conclude that the upstream flow deflections in these events are in accord with the current concept of the largescale structure of an ICME: a curved axial loop connected to the Sun, bounded by a curved (though not necessarily circular) cross section.
\end{abstract}

Key words. Interplanetary physics (flare and stream dynamics; interplanetary magnetic fields; interplanetary shocks)

\section{Introduction}

It is well known that interplanetary coronal mass ejections (ICMEs) undergo a significant interaction with the solar wind during their transit from the Sun to the Earth. The most obvious manifestation of this is their tendency to be decelerated or accelerated towards the speed of the ambient solar wind (e.g. Gopalswamy et al., 2000, 2001; Vršnak and Gopalswamy, 2002; Owens and Cargill, 2004). While the interaction between an ICME and the solar wind can be described in terms of an aerodynamic drag force and associated drag coef-

Correspondence to: $\mathrm{M}$. Owens

(mjowens@bu.edu) ficient (e.g. Cargill et al., 1995, 1996; Vršnak, 2001; Cargill, 2004), this in fact involves a number of complex stages.

When the ICME speed exceeds the relevant magnetohydrodynamic wave speed (usually the fast mode) a collisionless bow shock forms in front of the ICME that decelerates, compresses and heats the solar wind plasma. Behind this shock there is a sheath region of dense hot plasma and magnetic field that may be compressed and/or draped around the ICME. Indeed, magnetic field draping ahead of ICMEs has been directly observed in the outer heliosphere (McComas et al., 1988, 1989; Jones et al., 2002). The sheath is also where the shocked solar wind flow must be deflected around the ICME. The need for deflection arises from the fact that the solar wind plasma and field are frozen together, and thus cannot penetrate into the ICME. This is particularly clear for the case of magnetic clouds (e.g. Burlaga, 1988), where the ICME is treated as a large flux rope. Early work by Gosling et al. (1987) detected westward flow deflections with a typical magnitude of $\sim 25 \mathrm{~km} / \mathrm{s}$ and they concluded that ICMEs are systematically deflected eastward by the magnetic stresses of the Parker-spiral IMF acting on the west flank of ICMEs.

The magnitude and especially the direction of flow deflections measured by a spacecraft will depend on which part of the ICME the spacecraft encounters. This point will be expanded on in Sect. 3, but can be introduced here by considering a case where the ICME is a magnetic flux rope. The leading surface of such a flux rope has two radii of curvature: one due to the rooting of its footpoints at the Sun (axial curvature), and one due to its finite cross section (cross-sectional curvature). Since one would expect the deflected flows to be locally approximately parallel to the surface, the properties of the measured flows must reflect the ICME geometry.

It is thus clear that the detection of these deflected flows, and in particular a determination of their direction relative to the surface of the ICME, is an important diagnostic of the interaction between ICMEs and the solar wind. This paper presents an investigation of such flow deflections. In Sect. 2 we present a survey of non-radial flows in the solar wind at 

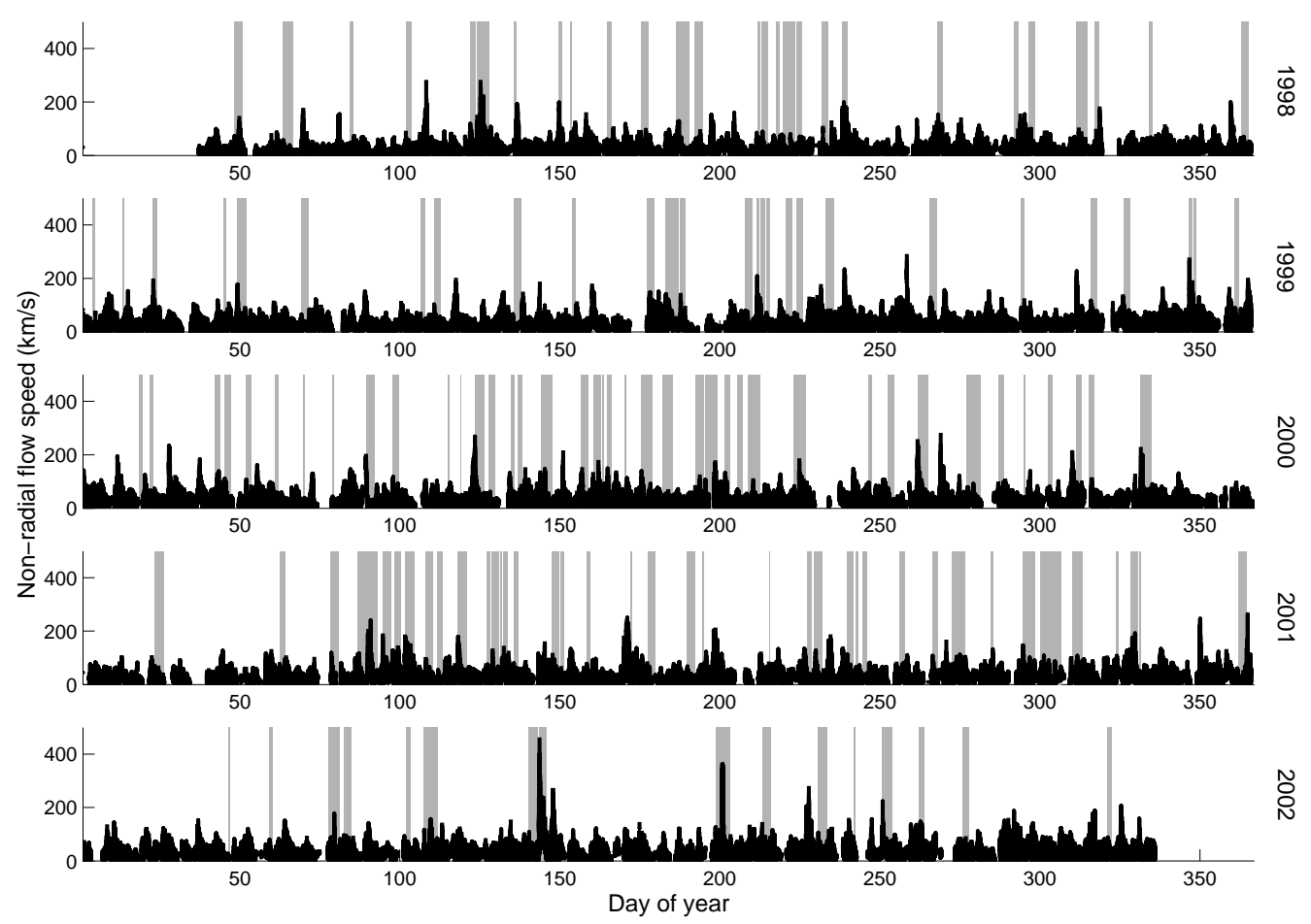

Fig. 1. The non-radial flow speed for 5 years of SWEPAM data. The grey shaded areas represent regions of solar wind identified as ICMEs by Cane and Richardson (2003).

$1 \mathrm{AU}$ using several years of interplanetary data, and determine what fraction can be associated with ICMEs. This provides a broad overview of the occurrence of flow deflections. In Sect. 3 we discuss how the flow deflection depends on the curvature of the leading surface of the ICME and present an analysis technique that can determine the flow deflection in the context of the spacecraft location with respect to the ICME. Section 4 presents a number of case studies of flow deflections.

\section{Survey of non-radial solar wind flows}

We first examine the existence of non-radial flows in the solar wind at $1 \mathrm{AU}$. If the magnitude of non-radial flows associated with ICMEs exceeds that in the ambient solar wind, then such flows are likely to be a signature of ICME-induced flow deflections. Solar wind plasma data from the SWEPAM instrument (McComas et al., 1998) on the Advanced Composition Explorer (ACE) spacecraft between 1 January 1998 and 31 December 2002 are used. We work in GSE coordinates, so that a radial ICME motion translates into motion in the $-\mathrm{x}_{G S E}$ direction. We then define the non-radial (or transverse) flow speed $\left(\left|\mathrm{V}_{t}\right|\right)$ as $\mathrm{V}_{t}^{2}=\mathrm{V}_{Y}^{2}+\mathrm{V}_{Z}^{2}$, where $\mathrm{V}_{Y}$ and $\mathrm{V}_{Z}$ are the components of the flow velocity in the $\mathrm{y}_{G S E}$ and $\mathrm{z}_{G S E}$ directions, respectively. We note that the survey results are very similar if the angle between the velocity vector and the radial direction is used in place of the non-radial flow speed.
The solid black lines in Fig. 1 shows the 5-min averaged $\left|\mathrm{V}_{t}\right|$ between 1998 and 2002. To establish the connection of $\left|\mathrm{V}_{t}\right|$ with ICMEs, we draw on the ICME survey of Cane and Richardson (2003). On the basis of magnetic field and plasma data, they identified 214 ICMEs at $1 \mathrm{AU}$ in the period 1996-2002 from which we use the subset of 180 ICMEs observed in 1998-2002. Periods of solar wind identified as ICMEs are shown as the grey shaded areas in Fig. 1. One can see at a glance that there appears to be some overlap between high non-radial velocities and the presence of ICMEs.

Noting that the average magnitude of the non-radial flow velocity in the solar wind for the period considered was $\sim 30 \mathrm{~km} / \mathrm{s}$, we can make things more concrete and define a non-radial flow (NRF) as an interval of solar wind with $\left|\mathrm{V}_{t}\right|>50 \mathrm{~km} / \mathrm{s}$. Multiple NRFs occurring within $12 \mathrm{~h}$ are presumed to be driven by the same disturbance, and are treated as a single event. Figure 2a shows a histogram of the maximum non-radial speed associated with all NRF events observed in the 5 years of SWEPAM data. The light shaded area indicates NRFs that occurred within $12 \mathrm{~h}$ of solar wind intervals identified as ICMEs by Cane and Richardson (2003), with the solid line showing the fraction (0 to $100 \%$ from top to bottom of plot) of NRFs in each speed bin associated with ICMEs. The dark shaded area and dashed line represent a subset of fast ICMEs, defined as having average radial speeds greater than $450 \mathrm{~km} / \mathrm{s}$. Approximately half of the larger $(>100 \mathrm{~km} / \mathrm{s})$ non-radial flows are readily associated with ICMEs. Indeed, both of the largest NRFs $(>300 \mathrm{~km} / \mathrm{s})$ are associated with fast ICMEs and from Fig. 1 appear to 

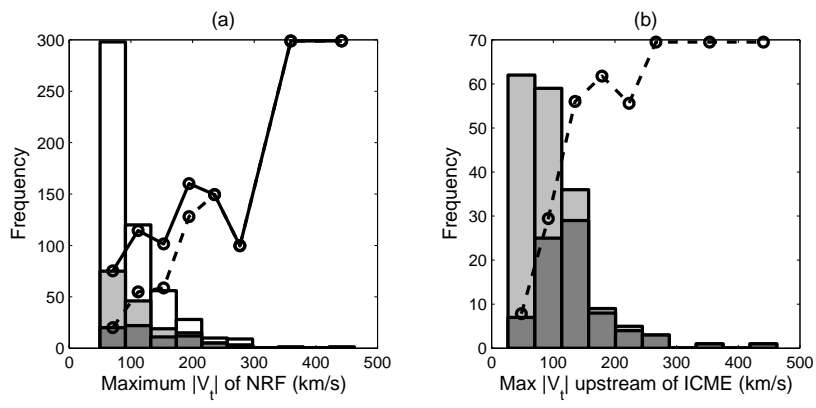

Fig. 2. Plot (a) shows a histogram of the maximum transverse flow speed of non-radial flows (NRFs) observed by ACE. We define a $\mathrm{NRF}$ as a period of solar wind with $\left|\mathrm{V}_{t}\right|>50 \mathrm{~km} / \mathrm{s}$. The light (dark) shaded area indicates NRFs that occurred within $12 \mathrm{~h}$ of solar wind intervals identified as (fast) ICMEs. The solid (dashed) line shows the percentage of NRFs in each speed bin associated with (fast) ICMEs. Plot (b) shows a histogram of the maximum transverse flows speeds upstream of ICMEs (dark regions indicate fast ICMEs, with the dashed line showing the percentage of all ICMEs that are fast). The upstream region is taken to be the 12 -h period ahead of the ICME leading edge.

occur at the boundaries between multiple ICMEs.

However, it is also clear from Figs. 1 and $2 \mathrm{a}$ that not all ICMEs generate significant solar wind flow deflections, and conversely, not all large non-radial flows can be readily associated with ICMEs. We thus took the identified ICMEs in the Cane and Richardson (2003) data set, and examined the magnitude of the upstream flow deflections. Here the upstream region is defined as the solar wind in the $12-\mathrm{h}$ period preceding the ICME leading edge. For each ICME, the maximum non-radial flow speed in this upstream region was found and the results are shown in Fig. 2b. The light and dark shaded areas represent all and fast ICMEs, respectively, with the dashed line showing the fraction (0 to $100 \%$ from top to bottom of the plot) of ICMEs in that non-radial speed bin that are fast. The mean value of the maximum transverse flow speed preceding all (fast) ICMEs is $101.3 \mathrm{~km} / \mathrm{s}(137.0 \mathrm{~km} / \mathrm{s})$, with $38 \%(65 \%)$ of all (fast) ICMEs exhibiting a maximum non-radial flow speed greater than $100 \mathrm{~km} / \mathrm{s}$ in the upstream solar wind region.

It is well known that a non-radial component to the solar wind velocity can be generated at the interface of two interacting solar wind streams. However, at least during periods close to solar maximum, a significant fraction of the nonradial solar wind flows at $1 \mathrm{AU}$ are associated with ICMEs. The deflection of the ambient solar wind by the transient ejecta suggests a means for estimating both part of the ICME encountered by the observing spacecraft, and the shape of the ICME leading edge. In the following section we outline a method for interpreting the upstream flow deflections in terms the large-scale structure of ICMEs.

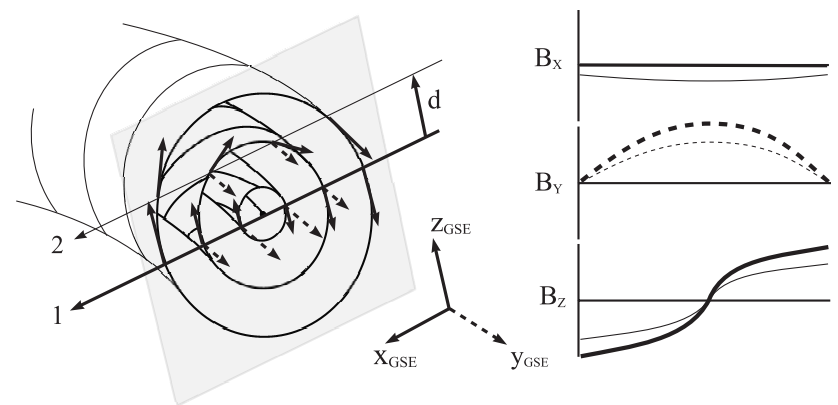

Fig. 3. A sketch of the geometry of an ICME. The local axis of the magnetic cloud is in the $\mathrm{y}_{G S E}$ direction, and the circular crosssection is in the $\mathrm{x}_{G S E}-\mathrm{z}_{G S E}$ plane. Vectors perpendicular (parallel) to $\mathrm{y}_{G S E}$ are shown as solid (dashed) lines. Two spacecraft trajectories through the flux-rope are shown, and sketches of the observed magnetic field parameters are shown on the right-hand side, with trajectory 1 (2) shown as the thick (thin) line.

\section{Analysis of flow deflections}

The results of Sect. 2 indicate the need to develop analysis methods to examine flow deflections at individual ICMEs. The magnitude and direction of the deflection must depend on the geometry of the leading surface of the ICME and we adopt the scenario that an ICME is a large magnetic flux rope, with both feet rooted in the solar surface (i.e. a magnetic cloud). Then in the absence of magnetic reconnection between the ICME and solar wind magnetic fields (for details see Cargill et al., 1996), the leading surface of the ICME is a curved flux surface through which plasma does not penetrate, implying a deflected flow parallel to this surface. The direction and magnitude of the deflected flow will depend on the orientation of the ICME surface and its speed relative to the solar wind.

The shape of this surface will be determined by two types of curvature: axial curvature due to the rooting of the footpoints of the ICME at the Sun and cross-sectional curvature due to its internal magnetic field structure. While each curvature is determined by different processes, there is some evidence that they have similar scales (Russell and Mulligan, 2002). An important issue is whether the local radii of curvature are the same at all points on the surface. Early work (e.g. Burlaga, 1988) suggested that magnetic clouds had a circular cross section, but more recent experimental and theoretical work indicates that ICMEs can be elongated considerably in a direction perpendicular to their direction of motion (Russell and Mulligan, 2002; Cargill and Schmidt, 2002). This nonuniformity of curvature can have important consequences.

As an example of the type of flow deflections expected, consider the schematic picture of a flux rope shown in Fig. 3. For simplicity we present the situation at the ICME nose where the flux rope axis and axial radius of curvature point in the $\mathrm{y}_{G S E}$ and $\mathrm{x}_{G S E}$ directions, respectively. Vectors perpendicular (parallel) to $\mathrm{y}_{G S E}$ are shown as solid (dashed) lines. If the spacecraft encounters the ICME at $\mathrm{y}_{G S E}=0$ and 

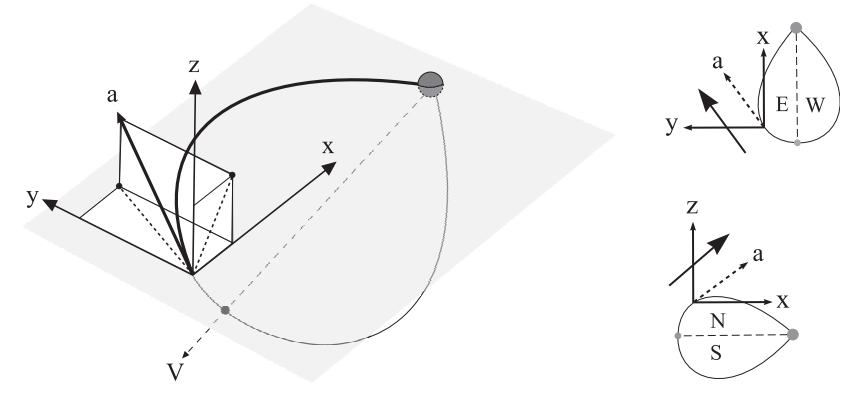

Fig. 4. An explanation of the axis projections in terms of the largescale orientation of magnetic clouds. The curved line represents a magnetic cloud axis, the dot represents the nose of the cloud (the effect of solar rotation has been ignored). The axis intersects the ecliptic plane (shown as the grey panel), where local axis orientation (a) is the thick arrow. Projections of the axis onto the ecliptic and $\mathrm{x}_{G S E^{-}} \mathrm{z}_{G S E}$ planes are shown as dashed lines, and are interpreted in terms of the ICME "flanks" in the two smaller diagrams on the right-hand side. By requiring the $\mathrm{x}_{G S E}$ component of the axis to be positive, a positive (negative) $\mathrm{y}_{G S E}$ indicates an intersection east (west) of the nose. Similarly, a positive (negative) $\mathrm{z}_{G S E}$ indicates an intersection north (south) of the nose.

a distance $d$ above the $\mathrm{z}_{G S E}=0$ axis, the flow must be deflected in the positive $\mathrm{z}_{G S E}$ and $\mathrm{x}_{G S E}$ directions. If $d$ is then replaced by $-d$, the deflection in the $\mathrm{z}_{G S E}$ direction is reversed. If the spacecraft is displaced in the $\mathrm{y}_{G S E}$ direction, but remains at $\mathrm{z}_{G S E}=0$, the deflection then acquires a component in the $\mathrm{y}_{G S E}$ direction. Finally, a displacement away from both $\mathrm{y}_{G S E}=0$ and $\mathrm{z}_{G S E}=0$ leads to a general deflected flow with components in all three directions. Thus, if one knows the (local) orientation of the flux rope axis, and whether one is above or below the mid-point of the ICME cross section, one can predict the direction of the non-radial flow. However, the magnitude and direction of the flow will also depend on the shape of the leading surface.

Turning first to the orientation of the ICME axis, this can be estimated using Minimum Variance Analysis (MVA, see Sonnerup and Cahill, 1967, for a general discussion and Bothmer and Schwenn, 1988, for application to ICMEs). For the case shown in Fig. 3, the maximum $\left(\mathbf{e}_{\max }\right)$, intermediate $\left(\mathbf{e}_{i n t}\right)$, and minimum $\left(\mathbf{e}_{\min }\right)$ variance directions of the magnetic field will be in the $\mathrm{z}_{G S E}, \mathrm{y}_{G S E}$ and $\mathrm{x}_{G S E}$ directions, respectively (Bothmer and Schwenn, 1998), subject to ambiguities discussed below. Note, in particular, that the axis orientation is determined by the intermediate variance direction. Sketches of the magnetic field components for this example are shown on the right-hand side of Fig. 3 for a spacecraft passing through the centre (thick lines) and a distance $d$ above the axis (the thin lines).

There is a $180^{\circ}$ ambiguity in the variance directions and it is therefore necessary to impose additional constraints. We require $\mathbf{e}_{i n t}$ to be parallel to the axial magnetic field (in this case to point in the positive $\mathrm{y}_{G S E}$ direction) and the minimum variance direction to have a positive $\mathrm{x}_{G S E}$ component. Thus, the right-handed variance coordinate system for the flux-rope shown in Fig. 3 would be $\left(\mathbf{e}_{\max }, \mathbf{e}_{\text {int }}\right.$, $\left.\mathbf{e}_{\text {min }}\right)=\left(-\mathrm{z}_{G S E}, \mathrm{y}_{G S E}, \mathrm{x}_{G S E}\right)$. In the variance coordinate system, the chirality of the magnetic cloud is defined by the sense of the rotation in the maximum variance magnetic field: the positive to negative rotation shown in the example indicates a left-handed rotation.

For "off-axis" crossings (trajectory 2 and thin lines in Fig. 3), the variance directions are not so well defined. This manifests itself in lower eigenvalue ratios, coupled with less recognisable signatures in the behaviour of the variance magnetic field components (the thin lines in Fig. 3). Thus we couple the results of MVA with a visual inspection of the magnetic field hodograms, and some judgement is required to establish the viability of MVA. The error in the axis orientation estimated by variance analysis increases with the increasing closest approach distance of the spacecraft to the axis $(|d|)$, but nominally this error is within $10^{\circ}$ (e.g. Burlaga and Behannon, 1982).

For crossings through the flux rope axis $\left(\mathrm{y}_{G S E}=0\right.$ here), the magnetic field in the minimum variance direction $\left(\mathrm{B}_{\min }\right)$ is approximately zero. However, for off-axis crossings, $\mathrm{B}_{\min } \neq 0$. This fact can be used to qualitatively infer the spacecraft position relative the axis (i.e. $d$ in Fig. 3), using the handedness of the flux-rope and the polarity of the $\mathrm{B}_{\min }$. For example, trajectory 2 in Fig. 3 sees a positive to negative rotation in $\mathrm{B}_{\max }$, indicating a left-handed flux-rope. It also measures a negative $\mathrm{B}_{\text {min }}$. One can then infer that the position of the spacecraft relative to the flux-rope axis $(d)$, has a negative value of $\mathbf{e}_{\text {max }}$. We term this type of ICME encounter as a "negative" crossing (conversely, a crossing at a position with a positive $\mathbf{e}_{\max }$ component would be termed a "positive" crossing). Note that as $\mathbf{e}_{\max }=-\mathrm{z}_{G S E}$ in this particular case, the "negative" crossing actually translates to a spacecraft interception "above" the axis relative to the $\mathrm{z}_{G S E}$ direction. However, it is logical to work in the variance coordinate system when defining the spacecraft crossing relative to the axis, as the axis may (in principle) have any orientation with respect to the GSE coordinate axes.

Based on the above discussion, we can write down general rules for the type of crossing. Defining the handedness $(\mathrm{H})$ to be $+(-) 1$ for right-(left-) handed flux ropes, the "crossing", as defined above, is given by the sign of $-\mathrm{H} \operatorname{sgn}\left(\mathrm{B}_{\min }\right)$ where $\operatorname{sgn}(\mathrm{x})$ is $1(-1)$ for $\mathrm{x}>(<) 0$. The crossing location relative to the $\mathrm{z}_{G S E}=0$ axis is given by the sign of $-\mathrm{Hsgn}\left(\mathrm{B}_{\min }\right) \operatorname{sgn}\left(\mathbf{e}_{\max }(\hat{\mathbf{z}})\right)$.

We proceed with the analysis of the flow deflection by defining a local axis vector $\hat{\boldsymbol{a}}$ to lie along $\mathbf{e}_{i n t}$, but to have a positive $\mathrm{x}_{G S E}$ component (i.e. $\hat{\boldsymbol{a}}$ is either parallel or antiparallel to $\mathbf{e}_{i n t}$ : see Fig. 4 for a sketch). This defines $\hat{\boldsymbol{a}}$ to point in the direction of the flow deflection (in the ICME rest frame) resulting from axial curvature. The "flank" of the ejecta encountered by the spacecraft can be inferred from the axis vector if the ICME is assumed to have the form of a curved axial loop rooted at both ends to the Sun, as shown in Fig. 4. To enable an intercomparison of ICME encounters, we classify ICMEs based upon axis projections onto the $\mathrm{x}_{G S E}-\mathrm{y}_{G S E}$ and $\mathrm{x}_{G S E}-\mathrm{z}_{G S E}$ planes. A positive (negative) 
$\mathrm{y}_{G S E}$ component indicates an intersection east (west) of the nose. Similarly, a positive (negative) $\mathrm{z}_{G S E}$ indicates an intersection north (south) of the nose.

We now assume that the leading edge of the ICME is locally planar. Thus, the normal to the local leading edge $(\hat{\mathbf{n}})$, the incoming flow velocity (directed along $\mathbf{x}_{G S E}$ ) and the direction of the deflected flow velocity $\left(\mathbf{V}_{d}\right)$ all lie in the same plane, such that: $\hat{\mathbf{V}}_{\mathbf{d}}=\hat{\mathbf{n}} \times\left(\hat{\mathbf{x}}_{G S E} \times \hat{\mathbf{n}}\right)$. For axial encounters (i.e. $d=0)$, cross-sectional curvature can be ignored, and the normal to the leading edge is given by: $\hat{\mathbf{n}}=\hat{\boldsymbol{a}} \times\left(\hat{\boldsymbol{a}} \times \hat{\mathbf{x}}_{G S E}\right)$. Thus, for spacecraft trajectories intersecting the axis, flow deflections should be axis-aligned, as shown by the solid arrows in the right-hand side of Fig. 4. (When $\hat{\boldsymbol{a}}$ is perpendicular to $\mathbf{x}_{G S E}$, the direction of the deflected flow is undefined. This is the stagnation point at the nose of the ejecta.)

Conversely, without axial curvature (i.e. a nose encounter) the flow will be deflected around the cross section of the ICME, perpendicular to $\hat{\boldsymbol{a}}$. We define a unit vector $\hat{\mathbf{c}}$ to lie in the plane of the leading edge, orthogonal to $\hat{\mathbf{a}}$ and to have a positive $\mathbf{x}_{G S E}$ component (see Fig. 5). Furthermore, we require that $\hat{\mathbf{c}}$ has a positive (negative) $\mathbf{e}_{\max }$ component for "positive" ("negative") spacecraft crossings, as defined above. Thus, $\hat{\mathbf{c}}$ points in the direction of the flow deflection resulting from cross-sectional curvature (see also Fig. 5). For spacecraft crossings near the nose of ejecta (where axial curvature can be ignored) the normal to the leading edge is given by: $\hat{\mathbf{n}}=\hat{\mathbf{c}} \times\left(\hat{\mathbf{c}} \times \hat{\mathbf{x}}_{G S E}\right)$. For spacecraft trajectories just above the axis relative to $\mathbf{e}_{\max }$ (i.e. the closest approach distance of the spacecraft to the axis is negligible), $\hat{\mathbf{c}} \rightarrow \mathbf{e}_{\text {max }}$, whereas $\hat{\mathbf{c}} \rightarrow \mathbf{e}_{\text {min }}$ for trajectories clipping the outer edge (i.e. the closest approach distance of the spacecraft to the axis is comparable to the radius of the flux-rope), as shown in Fig. 5.

In general, both axial and cross-sectional effects are important, so the normal to the leading edge is $\hat{\mathbf{n}}=\hat{\boldsymbol{a}} \times \hat{\mathbf{c}}$ and the direction of the flow deflection can then be written as: $\left.\hat{\mathbf{V}}_{\mathbf{d}}=(\hat{\mathbf{a}} \times \hat{\mathbf{c}}) \times\left[\hat{\mathbf{x}}_{G S E} \times(\hat{\boldsymbol{a}} \times \hat{\mathbf{c}})\right]\right)$. Thus, the deflected flow direction always lies between the vectors $\hat{\boldsymbol{a}}$ and $\hat{\mathbf{c}}$. Variance analysis can completely describe the axial vector $\hat{\boldsymbol{a}}$. However, without the use of a flux-rope model (and hence assumptions about the cross-sectional shape of ICMEs), we are unable to quantitatively estimate the closest approach distance $(d)$, and therefore are unable to completely describe $\hat{\mathbf{c}}$. For "positive" ("negative") axis crossings, our knowledge of $\hat{\mathbf{c}}$ is limited to the fact that it must always lie between $\mathbf{e}_{\max }$ and $\mathbf{e}_{\min }\left(-\mathbf{e}_{\max }\right.$ and $\left.\mathbf{e}_{\min }\right)$. Hence, we know the orientation of $\hat{\mathbf{c}}$ only to within $90^{\circ}$. For a "positive" magnetic cloud encounter (i.e. above the axis relative to $\mathbf{e}_{\max }$ ) the upstream flow deflection should lie between $\hat{\boldsymbol{a}}$ and $\mathbf{e}_{\min }$, and have a positive $\mathbf{e}_{\text {max }}$ component, whereas for "negative" crossings, $\mathbf{V}_{d}$ is again expected to lie between $\hat{\boldsymbol{a}}$ and $\mathbf{e}_{\text {min }}$, but with a negative $\mathbf{e}_{\text {max }}$ component.

Finally, in order to apply the proposed analysis technique it is necessary to transform the measured velocities (i.e. in the spacecraft rest frame) into the rest-frame of the ICME, so that the solar wind flows toward the leading edge of the ICME in the positive $\mathrm{x}_{G S E}$ direction. However, there often exist large velocity gradients through a radial cut of an ICME

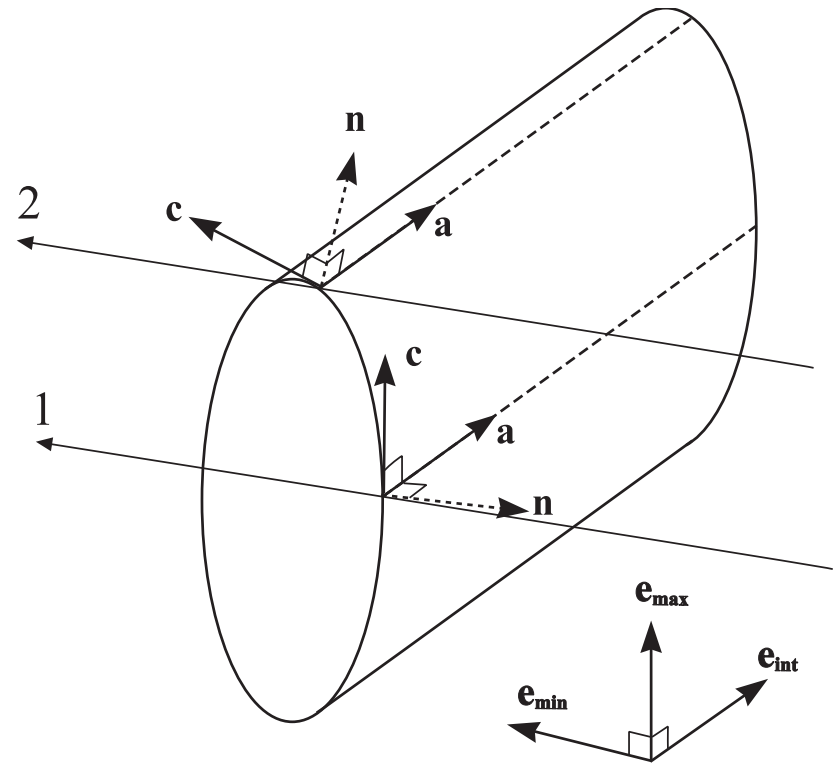

Fig. 5. The normals to the leading edge of an ICME for different closest approach distances of the spacecraft to the axis: trajectory 1 passes close to the axis, whereas trajectory 2 clips the outer edge of the ICME cross section. For trajectory $1, \mathrm{c} \rightarrow \mathrm{e}_{\max }$, resulting in $\mathrm{n} \rightarrow-\mathrm{e}_{\min }$. For trajectory $2, \mathrm{c} \rightarrow \mathrm{e}_{\min }$, resulting in $\mathrm{n} \rightarrow \mathrm{e}_{\max }$.

owing to its expansion. Additionally, matters are complicated if the expansion of the ICME is not completely cylindrically symmetric, meaning identification of the characteristic ICME speed, and hence performing the correct frame transformation, is non-trivial.

In this study we limit the analysis of the flow deflection to the non-radial components of the flow, avoiding the need for any transformation. This restricts comparison between the expected and observed flows to projections onto the nonradial (i.e. $\mathrm{y}_{G S E}-\mathrm{z}_{G S E}$ ) plane. In the case studies of Sect. 4, we use the angle between the upstream flow direction and the axis vector, defined by $\cos \theta=\left|\mathbf{V}_{d} \cdot \mathbf{e}_{\text {int }}\right| /\left|\mathbf{e}_{\text {int }}\right|\left|\mathbf{V}_{d}\right|$, where only the $\mathrm{y}$ and $\mathrm{z}$ components of the vectors are used. However, the effect of projection must be taken into consideration. For spacecraft encounters through the ICME axis, $\mathbf{V}_{d}$ is expected to lie along the non-radial projection of $\hat{\boldsymbol{a}}$, whereas for encounters at the very top or bottom edge of the ICME, $\mathbf{V}_{d}$ should be aligned with the projection of $\mathbf{e}_{\min }$. At intermediate distances from the axis, $\mathbf{V}_{d}$ should thus lie between the projections of $\hat{\boldsymbol{a}}$ and $\mathbf{e}_{\min }$, with a positive (negative) $\mathbf{e}_{\max }$ component for "positive" ("negative") spacecraft crossings. Thus, the position of the observed flow deflection between the two extremes allows for a first-order estimate of the distance from the axis at which the spacecraft intercepts the ICME. To quantify this parameter we calculate the ratio of $\theta$ to the angle between the projections of $\hat{\boldsymbol{a}}$ and $\mathbf{e}_{\text {min }}$, the latter angle measured so as to include a positive (negative) $\mathbf{e}_{\max }$ component for "positive" ("negative") spacecraft crossings. The angle ratio is denoted $\theta_{n}$, and is expected to have a value between 0 (for axis aligned flows) and 1 (for $\mathbf{e}_{\min }$ aligned flows). 
Table 1. The five magnetic clouds with ordered non-radial flows in the upstream sheath region. The ICME number indicates the event number in the Cane and Richardson (2003) ICME catalogue.

\begin{tabular}{ccccccc}
\hline Event & ICME number & Year & ICME Start (DOY) & ICME End (DOY) & Sheath start (DOY) & Sheath end (DOY) \\
\hline A & 35 & 1998 & 63.62 & 65.08 & 63.45 & 63.6 \\
B & 57 & 1998 & 268.3 & 269.4 & 267.97 & 268.26 \\
C & 136 & 2000 & 277.65 & 279.0 & 277.01 & 277.42 \\
D & 154 & 2001 & 102.35 & 103.29 & 101.55 & 101.92 \\
E & 186 & 2001 & 304.87 & 306.34 & 304.54 & 304.82 \\
\hline
\end{tabular}

Table 2. Details of the flux-rope orientations and upstream flow deflections for the 5 case studies considered. Orientation parameters: the three variance directions in $\left(\mathrm{x}_{G S E}, \mathrm{y}_{G S E}, \mathrm{z}_{G S E}\right)$ format, maximum to intermediate and intermediate to minimum eigenvalue ratios, the direction of the axis vector relative to $\mathbf{e}_{i n t}$, magnetic field strength in the $\mathbf{e}_{\min }$ direction $\left(<\mathrm{B}_{\min }>\right)$ and its fractional contribution to the total field strength $\left(<\left|\mathrm{B}_{\min }\right|>/<|\mathbf{B}|>\right)$. The average flow in the upstream disturbance $(\mathbf{V})$ is listed as $\left(\mathrm{V}_{X}, \mathrm{~V}_{Y}, \mathrm{~V}_{Z}\right)$, in GSE coordinates.

\begin{tabular}{ccccccccc}
\hline & $\mathbf{e}_{\text {max }}$ & $\mathbf{e}_{\text {int }}$ & $\mathbf{e}_{\text {min }}$ & $\begin{array}{c}\lambda_{\max } / \lambda_{\text {int }}, \\
\lambda_{\text {int }} / \lambda_{\min }\end{array}$ & $\mathbf{\hat { \mathbf { a } }}$ & $\begin{array}{c}<\mathrm{B}_{\min }> \\
(\mathrm{nT})\end{array}$ & $\begin{array}{c}<\left|\mathrm{B}_{\min }\right|>/ \\
<|\mathbf{B}|>\end{array}$ & $\mathbf{V}(\mathrm{km} / \mathrm{s})$ \\
\hline $\mathrm{A}$ & $(0.19,0.45,-0.87)$ & $(-0.53,0.80,0.30)$ & $(0.83,0.40,0.39)$ & $11.5,5.1$ & $-\mathbf{e}_{\text {int }}$ & $0.98 \pm 0.77$ & 0.09 & $-(381,41,36)$ \\
$\mathrm{B}$ & $(0.21,0.97,-0.11)$ & $(-0.59,0.22,0.78)$ & $(0.78,-0.10,0.62)$ & $14.5,4.8$ & $-\mathbf{e}_{\text {int }}$ & $-6.6 \pm 1.2$ & 0.46 & $-(806,135,-33)$ \\
$\mathrm{C}$ & $(-0.34,0.27,-0.9)$ & $(0.38,0.92,0.13)$ & $(0.86,-0.29,-0.42)$ & $15.6,2.9$ & $\mathbf{e}_{\text {int }}$ & $2.8 \pm 2.6$ & 0.19 & $-(463,12,-74)$ \\
$\mathrm{D}$ & $(0.10,0.85,-0.53)$ & $(-0.32,0.53,0.79)$ & $(0.94,0.09,0.32)$ & $7.4,5.3$ & $-\mathbf{e}_{\text {int }}$ & $4.1 \pm 0.8$ & 0.45 & $-(677,53,-157)$ \\
$\mathrm{E}$ & $(0,0.03,-1)$ & $(0.16,0.99,0.03)$ & $(0.99,-0.16,0)$ & $6.9,7.8$ & & $0.9 \pm 1.3$ & 0.09 & $-(366,80,16)$ \\
\hline
\end{tabular}

\section{Examples of ICME-related flow deflections}

We now use the analysis developed in the previous section to compare the orientations of ICMEs with the associated flow deflections. This analysis is performed for five events, all observed with the ACE spacecraft, and documented in the Cane and Richardson (2003) catalogue. Of the 214 ICMEs listed in the catalogue, 54 are listed as "magnetic clouds", however 20 of these ICMEs occurred before ACE became operational. For the remaining 34 magnetic clouds we performed variance analysis on ACE magnetic field data (Smith et al., 1998). The event boundaries listed in the ICME catalogue are used as a reference point, but we vary the interval considered so as to obtain the required signatures in the variance directions, and to a lesser extent, maximise the eigenvalue ratios. It was possible to obtain satisfactory MVA axes orientations for 21 clouds using both a formal analysis and an inspection of the hodograms.

We next examined the flow deflections in the upstream solar wind for these events. For three events there was no identifiable disturbance, but in general the non-radial flow velocity in the sheath region ahead of the magnetic clouds was highly structured, containing discontinuities and gradual rotations. Thus, for the majority of the events it is not immediately clear how to define the deflected flow velocity. For this reason we restrict this study to events with a relatively constant non-radial flow velocity throughout the sheath. We identified 5 such events with sheath and ICME properties in Table 1.
The first example was of a fast magnetic cloud that occurred on day 63, 1998. Figure 6 shows ACE magnetic field and plasma observations of this event, with the magnetic field presented in the minimum, intermediate and maximum variance directions, which are in turn listed in Table 2, along with the eigenvalue ratios. The disturbance onset and ICME start and end times are shown by the solid vertical lines. The MVA analysis gives the most significant results when the ICME boundaries are taken at day numbers 63.62 and 65.08.

Figure 7 shows the variance vectors and $\hat{a}$ in a threedimensional representation (upper left), and projected onto the three planes of the GSE coordinate system (remaining panels). The magnetic field shows the characteristic positive to negative profile of the maximum variance magnetic field of a left-handed flux-rope. The average value of the minimum variance magnetic field is $\left\langle\mathrm{B}_{\min }>=0.98 \pm 0.77 \mathrm{nT}\right.$ (Table 2). The fraction of the ICME magnetic field strength in the minimum variance direction (i.e. $<\left|\mathrm{B}_{\min }\right|>|<| \mathbf{B} \mid>$ ) is very small (0.09), suggesting a crossing close to the axis. The positive value of $<\mathrm{B}_{\min }>$ for a left-handed flux-rope indicates the spacecraft trajectory was a "positive" crossing, in that the point of closest approach of the spacecraft to the axis has a positive $\mathbf{e}_{\max }$ component, but with a crossing below the $\mathrm{z}_{G S E}=0$ plane. The spacecraft encountered the south and west flanks of the ICME, so that $\hat{\boldsymbol{a}}=-\mathbf{e}_{i n t}$, as shown in Fig. 7.

The maximum 5 -min average non-radial flow speed is $55.3 \mathrm{~km} / \mathrm{s}$ and occurs approximately $75 \%$ of the way into the sheath. We note that this maximum flow is fairly representative of the transverse flow velocity throughout 

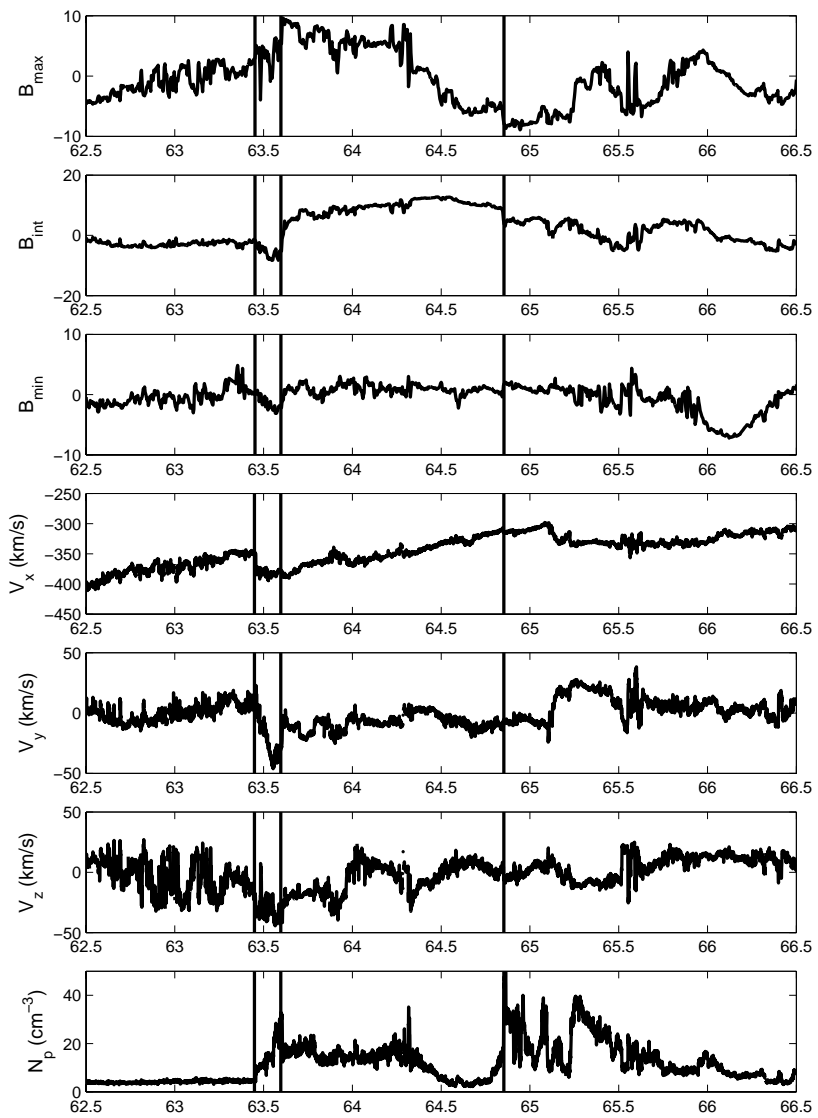

Fig. 6. Event A, day 63 1998. Magnetic field data (magnetic field in variance coordinates) is shown in the top 3 panels, ion data $\left(\mathrm{V}_{X}\right.$, $\mathrm{V}_{Y}$ and $\mathrm{V}_{Z}$ components of the proton velocity, and proton density) are shown in the bottom 4 panels. The disturbance, ICME start and end times are shown by the solid vertical lines. There is a small non-radial flow in the sheath preceding the ICME.

the whole sheath region, wherein $\left\langle\mathrm{V}_{Y}\right\rangle=-22.3$ and $<\mathrm{V}_{Z}>=-31.4 \mathrm{~km} / \mathrm{s}$. The sheath velocity vector is shown in the final column of Table 2, and Fig. 7 shows the projections of the variance directions and upstream flow deflection onto the $\mathrm{y}_{G S E}-\mathrm{z}_{G S E}$ plane. Both the maximum and average observed transverse velocities in the sheath region agree with the flows expected for this ICME orientation.

As noted in Sect. 3, the direction of the flow deflection is expected to lie between $\hat{\boldsymbol{a}}$ and $\mathbf{e}_{\min }$. The "positive" crossing means that $\mathbf{V}_{d}$ should also have a positive $\mathbf{e}_{\max }$ component, as is the case. The angle $(\theta)$ between the maximum (average) deflected flow direction and the axis vector is $20.9^{\circ}\left(34.3^{\circ}\right)$. Correcting for projection (i.e. dividing by $203.8^{\circ}$, the angle between $\hat{\boldsymbol{a}}$ and $\mathbf{e}_{\min }$, and rotating through positive $\mathbf{e}_{\max }$ ) gives $\theta_{n}$ of 0.10 and 0.17 for the maximum and average flow vectors, respectively. Thus, the close approach to the axis suggested by the small minimum variance magnetic field is supported by the small angle between the flow deflection and axis, as projected onto the non-radial plane.
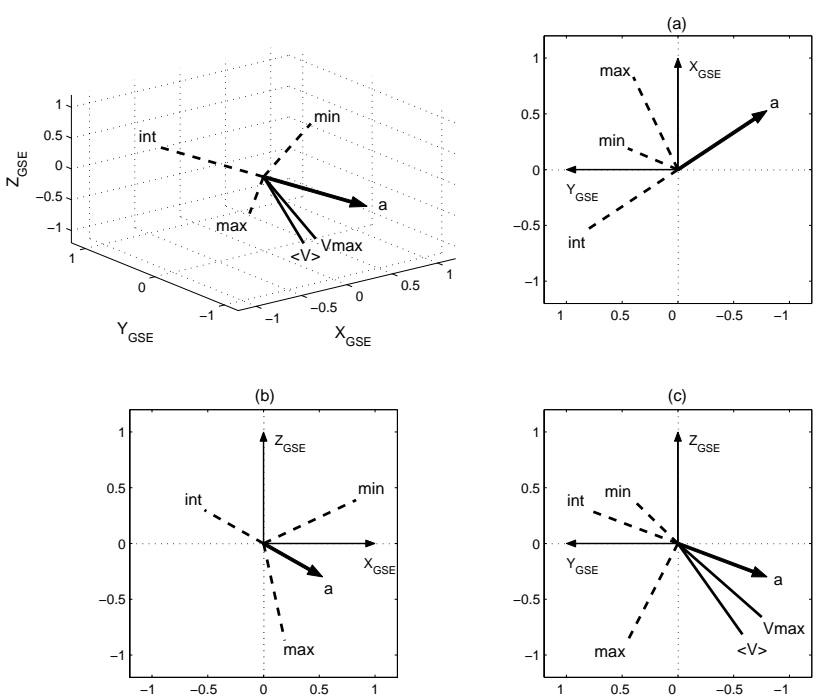

Fig. 7. The top left panel shows the axis orientation, variance directions and flow deflections of event A. Plot (a) shows the projections onto the ecliptic plane, plot (b) shows the projections onto the $\mathrm{x}_{G S E}{ }^{-\mathrm{Z}_{G S E}}$ plane. These projections should be compared to Fig. 4. The spacecraft encountered the south and west flanks of the ICME. Plot (c) shows flow deflections and variance directions of event $\mathrm{A}$ projected onto the non-radial (i.e. $\mathrm{y}_{G S E}-\mathrm{z}_{G S E}$ ) plane. Variance directions are shown as dashed lines, the axis vector is the solid arrow. The maximum and average transverse sheath velocity unit vectors are shown as solid lines. The observed flow deflections are consistent with the "positive" crossing inferred by variance analysis (i.e. they both lie between the axis and minimum variance directions, rotating through positive $\mathbf{e}_{\max }$ ).

The details of the remaining four events are summarised in Fig. 8 where the projection of the variance vectors and $\mathbf{V}_{d}$ on the y-z plane are shown, as well as in Tables 1 and 2. For each event, we find the following:

- Event B is a well-defined magnetic cloud and has a positive to negative rotation of $\mathrm{B}_{\max }$, indicative of a lefthanded rotation of the flux-rope magnetic field, and is a "negative" traversal. The minimum variance magnetic field accounts for a significant fraction of the total magnetic field of the ICME, suggesting that the distance of closest approach to the axis was much larger than for event A. The spacecraft encountered the south and west flanks of the ICME so that $\hat{\boldsymbol{a}}=-\mathbf{e}_{\text {int }}$. There is a large non-radial flow in the sheath region with a maximum 5 -min averaged speed of $139 \mathrm{~km} / \mathrm{s}$, occurring $78 \%$ of the way through the sheath. The average nonradial flow components over the duration of the sheath are $\left\langle\mathrm{V}_{Y}\right\rangle=-55.6$ and $\left\langle\mathrm{V}_{Z}\right\rangle=-1.3 \mathrm{~km} / \mathrm{s}$. When the velocity and variance directions are projected onto the non-radial plane (Fig. 8, top left panel), the observed flow deflection falls within the range predicted by the orientation and point of interception of the magnetic cloud. The maximum (average) upstream flow makes an 


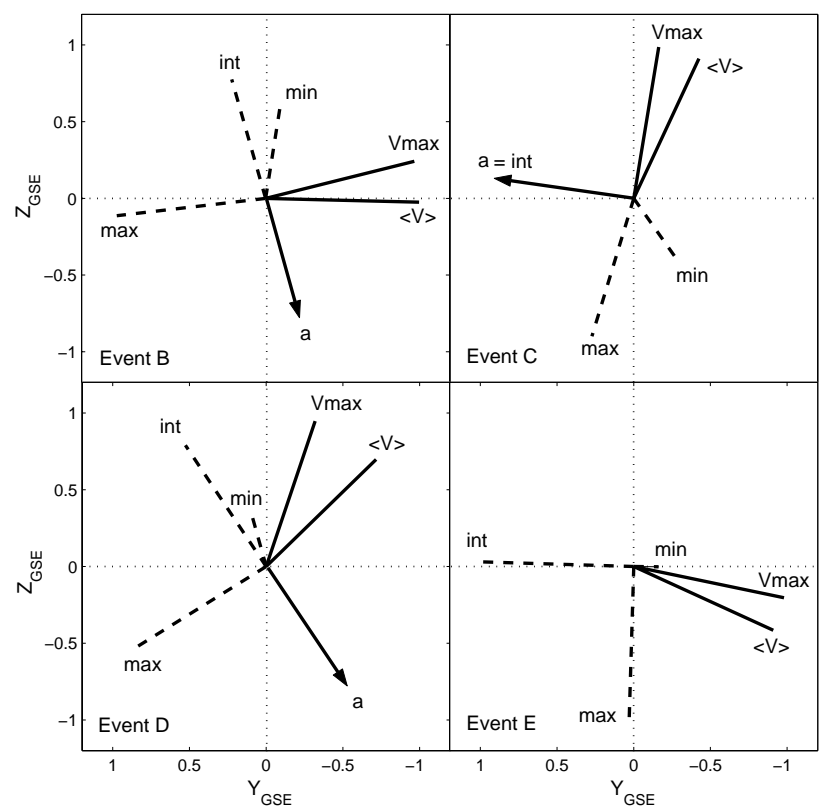

Fig. 8. The axis orientations, variance directions and flow deflections of events B, C, D and E, in the same format as Fig. 7c.

angle of $88.1^{\circ}\left(72.7^{\circ}\right)$ with the axis vector. Dividing by $106.4^{\circ}$ (the angle between $\hat{\boldsymbol{a}}$ and $\mathbf{e}_{\min }$ rotating through negative $\mathbf{e}_{\max }$ ) gives $\theta_{n}$ values of 0.83 and 0.68 using maximum and average upstream flows, respectively. We note the larger angle between the axis and flow vector projections compared with event A. This is also consistent with the closest approach being quite distant from the axis.

- Event $C$ has a smaller relative speed than in the previous two examples, and as a result the upstream disturbance takes the form of a bow-wave that has not steepened into a shock front. Nevertheless, the same deflection of flow should still occur at the leading edge. In the maximum variance direction, the magnetic field rotates smoothly from negative to positive values, indicating a right-handed flux-rope. In this case, $\hat{\boldsymbol{a}}=\mathbf{e}_{\text {int }}$, meaning ACE encountered the north and east flanks of the magnetic cloud. The maximum non-radial flow speed in the sheath is $75.3 \mathrm{~km} / \mathrm{s}$, occurring $72 \%$ of the way through the sheath. The $\mathrm{y}_{G S E}$ and $\mathrm{z}_{G S E}$ velocity components averaged over the sheath duration were -15.4 and $34.1 \mathrm{~km} / \mathrm{s}$, respectively. The orientation of the ICME compared with the upstream flow deflection is shown in Fig. 8 (top right panel). The observed flow deflection projected onto the non-radial plane lies between the axis and minimum variance direction, with a negative maximum variance component, consistent with the orientation and point of observation estimated by variance analysis. The value of $\theta_{n}$ for the maximum (average) upstream flow direction is $0.40(0.47)$, suggesting a closest approach further from the axis than event A, but closer than event $\mathrm{B}$. The fraction of the magnetic

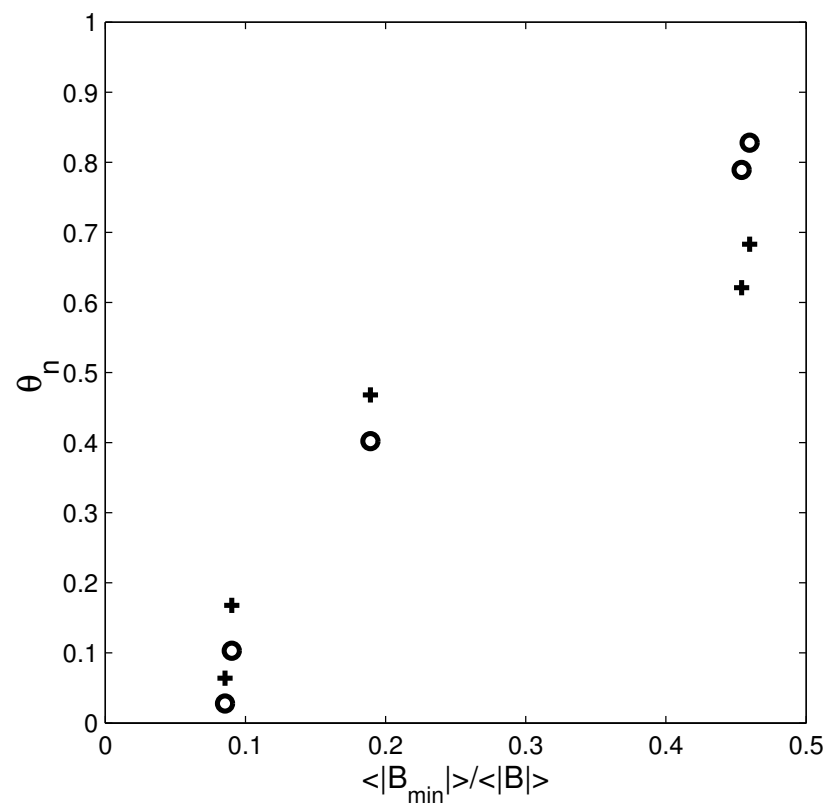

Fig. 9. The $\theta_{n}$ value (i.e. the "normalised" angle between the upstream flow direction and axis vector) as a function of normalised magnetic field strength in the minimum variance direction, for the 5 events considered. Circles (crosses) show $\theta_{n}$ calculated from the maximum (average) upstream flow direction.

field strength in the minimum variance direction agrees with this interpretation.

- Event D is an example with a strong non-radial flow ahead of the fast moving magnetic cloud. It is also interesting to note the presence of a large amplitude but short duration NRF at the trailing edge of the magnetic cloud, the result of a high speed stream behind the ICME. The flux-rope is right-handed, with a "negative" spacecraft crossing. The axis vector, $\hat{\boldsymbol{a}}=-\mathbf{e}_{i n t}$ here, indicating a south and west flank encounter. Averaging over the entire sheath gives $\left\langle\mathrm{V}_{Y}\right\rangle=-49.7 \mathrm{~km} / \mathrm{s}$ and $\left\langle\mathrm{V}_{Z}\right\rangle=48.4 \mathrm{~km} / \mathrm{s}$. Again, we find the upstream flow deflections are as expected for the orientation of the ICME and point of observation of the spacecraft. The flow vector is closer to the minimum variance direction than the axis vector: $\theta_{n}$ for the maximum (average) upstream flow direction is 0.79 (0.62), suggesting the closest approach of the spacecraft was a significant distance from the magnetic cloud axis. This is in accord with the large magnetic field strength in the minimum variance direction.

- Event E has a clear non-radial flow in the sheath region, flowed by a strong magnetic cloud signature. There is a SWEPAM data gap for much of the ICME body, but this does not affect our analysis of this event. Here the $\mathrm{x}_{G S E}$ and $\mathrm{z}_{G S E}$ components of the intermediate variance direction are very small, resulting in undetermined flank encounters, and hence a $180^{\circ}$ ambiguity in the axis 
vector. Examination of the magnetic field in the maximum variance direction reveals a left-handed flux-rope, and the small minimum variance field suggests a possible "positive" spacecraft crossing, though the small value of $\left\langle\mathrm{B}_{\min }\right\rangle$ is somewhat inconclusive. Thus, the point of interception of the spacecraft is likely to be close to the nose of the ejecta in both an axial and crosssectional sense. In the sheath region, the maximum 5min averaged transverse flow speed is $81.4 \mathrm{~km} / \mathrm{s}$, occurring $67 \%$ of the way through the sheath, and averaging over the whole sheath gives $\left\langle\mathrm{V}_{Y}\right\rangle=-52.5 \mathrm{~km} / \mathrm{s}$ and $\left\langle\mathrm{V}_{Z}\right\rangle=-24.0 \mathrm{~km} / \mathrm{s}$. Figure 8 (bottom right panel) shows the variance and the upstream flow directions projected onto the non-radial plane. The flow deflection is in agreement with a spacecraft crossing above the axis. We do not show an axis vector due to the ambiguity in the ICME flank encountered. However, the flow deflection suggests ACE intercepted the ICME west of the nose. Assuming $\hat{\boldsymbol{a}}=-\mathbf{e}_{\text {int }}$ (i.e. the west flank crossing suggested by the flow direction), $\theta_{n}$, using the maximum (average) $\mathbf{V}_{d}$, is $0.03(0.06)$, as expected for the small closest approach distance inferred by the negligible magnetic field strength in the minimum variance direction.

These results are summarised in Fig. 9 which shows $\theta_{n}$ plotted as a function of the flux-rope magnetic field strength in the minimum variance direction for the 5 events considered, with circles (crosses) showing $\theta_{n}$ calculated from the maximum (average) upstream flow direction. Both $\theta_{n}$ and $<\left|\mathbf{B}_{\text {min }}\right|>|<| \mathbf{B} \mid>$ are expected to increase with increasing distance from the axis of an ICME, though not necessarily linearly. These two independent methods of estimating the closest approach of the observing spacecraft to the axis of a magnetic cloud show good agreement. We also note that for these 5 events, the magnitude to the NRF speed in the sheath scales well with the ICME speed relative to the upstream solar wind.

\section{Discussion and conclusions}

This paper has addressed the plasma flows induced in the solar wind by the motion of ICMEs, in particular flows in the non-radial direction that must arise as fast-moving ICMEs push solar wind plasma aside. A survey of the non-radial flow speed in five years of solar wind data found approximately half of the large NRFs to be associated with ICMEs. Fast ICMEs were shown to drive the strongest transverse solar wind flows. The direction of the non-radial deflections was shown to be consistent with the local part of the ICME edge being encountered for five events studied in detail. However, it should be noted that the ordered flow deflections required to perform such analysis are relatively rare.

For spacecraft interceptions through the axis of a magnetic cloud, the minimum variance magnetic field should vanish. Furthermore, the upstream deflected flow should be axis aligned. As the closest approach distance between the spacecraft and axis increases, the magnetic field strength in the minimum variance direction should increase, and the deflected flow should rotate away from the axis toward the minimum variance direction. The 5 events considered in this study were consistent with these general trends (as shown in Fig. 9), though further observations are required to quantify this effect. Upstream flow deflections (in conjunction with modelling of the flux-rope magnetic field) provide a possible means to infer the cross-sectional shape and extent of ejecta, and will form the basis of a future study.

Finally, we note the existence of significant non-radial flows in the body of ejecta, though the magnitude of such flows are nominally less than the preceding sheath region. Further study of the sense of these flows coupled with the ICME flank encountered is required to ascertain whether the systematic eastward deflection of ICMEs reported by Gosling et al. (1987) is present in the ACE data set.

Acknowledgements. M. Owens thanks PPARC and QuintiQ for financial support. We have benefited from the availability of ACE data at NSSDC, in particular the MAG (N. Ness) and SWEPAM (D. McComas) instruments. We would also like to thank A. Rees for useful discussions.

The Editor in Chief thanks B. Vršnak for his help in evaluating this paper.

\section{References}

Bothmer, V. and Schwenn, R.: The structure and origin of magnetic clouds in the solar wind, Ann. Geophys., 16, 1, 1998.

Burlaga, L. F.: Magnetic clouds: Constant alpha force-free configurations, J. Geophys. Res., 93, 7217-, 1988.

Burlaga, L. F. and Behannon, K. W.: Magnetic clouds: Voyager observations between 2 and 4 AU, Sol. Phys., 81, 181, 1982.

Cane, H. V. and Richardson I. G.: Interplanetary coronal mass ejections in the near-Earth solar wind during 1996-2002, J. Geophys. Res., 108, 1156, 2003.

Cargill, P. J.: On the Aerodynamic Drag Force Acting on Coronal Mass Ejections, Solar Phys., 221, 135, 2004.

Cargill, P. J. and Schmidt, J. M.: Modelling interplanetary CMEs using magnetohydrodynamic simulations, Ann. Geophys., 20, 879, 2002.

Cargill, P. J., Chen, J., Spicer, D. S., and Zalesak, S. T.: Geometry of interplanetary magnetic clouds, Geophys. Res. Lett., 22, 647, 1995.

Cargill, P. J., Chen, J., Spicer, D. S., and Zalesak, S. T.: MHD simulations of the motion of magnetic flux tubes through a magnetized plasma, J. Geophys. Res., 101, 4855, 1996.

Gopalswamy, N., Lara, A., Lepping, R. P., Kaiser, M. L., Berdichevsky, D., and St. Cyr, O. C.: Interplanetary acceleration of coronal mass ejections, Geophys. Res. Lett., 27, 145, 2000.

Gopalswamy, N., Lara, A., Yashiro, S., Kaiser, M. L., and Howard, R. A.: Predicting the 1-AU arrival times of coronal mass ejections, J. Geophys. Res., 106, 29 207, 2001.

Gosling, J. T., Thomsen, M. F., Bame, S. J., and Zwickl, R. D.: The eastward deflection of fast coronal mass ejecta in interplanetary space, J. Geophys. Res., 92, 12 399, 1987.

Jones, G. H., Rees, A., Balogh, A., and Forsyth, R. J.: The draping of heliospheric magnetic fields upstream of coronal mass ejecta, Geophys. Res. Lett., 29, 1520, 2002. 
McComas, D. J., Gosling, J. T., Winterhalter, D., and Smith, E. J.: Interplanetary magnetic field draping about fast coronal mass ejections in the outer heliosphere, J. Geophys. Res. , 93, 2519, 1988.

McComas, D. J., Gosling, J. T., Bame, S. J., Smith, E. J., and Cane, $\mathrm{H}$. V.: A test of magnetic field draping induced $\mathrm{B}_{z}$ perturbations ahead of fast coronal mass ejecta, J. Geophys. Res., 94, 1465, 1989.

McComas, D. J., Bame, S. J., Barker S. J., Feldman, W. C., Phillips, J. L., Riley, P., and Griffee, J. W.: Solar wind electron proton alpha monitor (SWEPAM) for the Advanced Composition Explorer, Space Sci. Rev., 86, 563, 1998.

Owens, M. J. and Cargill, P. J.: Predictions of the arrival time of Coronal Mass Ejections at $1 \mathrm{AU}$ : An analysis of the causes of errors, Ann. Geophys., 22, 661, 2004.
Russell, C. T. and Mulligan, T.: On the magnetosheath thicknesses of interplanetary coronal mass ejections, Planet. Space Sci., 50, 527, 2002.

Smith, C. W., L'Heureux, J., Ness, N. F., Acuna, M. H., Burlaga, L. F., and Scheifele, J.: The ACE magnetic fields experiment, Space Sci. Rev., 86, 613, 1998.

Sonnerup, B. U. O. and Cahill, L. J.: Magnetopause structure and attitude from Explorer 12 observations, J. Geophys. Res., 72, 171, 1967.

Vršnak, B.: Dynamics of solar coronal eruptions, J. Geophys. Res., 106, 25 249, 2001.

Vršnak, B. and Gopalswamy, N.: Influence of aerodynamic drag on the motion of interplanetary ejecta, J. Geophys. Res., 107, 1029, 2002. 